The Impact of Large-Scale Surveys on Pulsating Star Research ASP Conference Series, Vol. 203, 2000

L. Szabados of D. W. Kurtz, eds.

\title{
Light Curve Analysis of Tycho Variable Stars
}

\author{
Ivan L. Andronov ${ }^{1,2}$, Jan Cuypers ${ }^{3,2}$, Sandrine Piquard ${ }^{2}$
}

Abstract. A variety of methods has been applied to the Tycho suspected variable stars to find reliable periods.

\section{Objective and Preparation of the Data}

Our aim was to study the Tycho suspected variable stars of the list of Piquard et al. (2000). For that purpose, a large variety of period analysis methods was applied, and a sample of 320 stars with known periods was used to evaluate their efficiencies. First and foremost, the Tycho observations were prepared in order to improve their reliability: the data were corrected for systematic errors (Halbwachs 1997), and they were averaged when the time interval was shorter than 1 min. The merged observations received weights depending on their accuracy estimates.

\section{Period Search: Approach I}

We adapted the PDM method (Stellingwerf 1978) taking into account the unequal weights of the data. The periodograms have been computed separately for the $B_{T}, V_{T}$ and $T$ bands using the $(5 \times 5)$ bin structure $(10 \times 2$ for eclipsing variables). A frequency was added to the list of possible candidates, if its "false alarm" probability due to noise was less than 0.002 . The phase diagrams corresponding to candidate periods were fitted by a trigonometric polynomial. The "best" periods correspond to the true period, or a multiple for one or more colors, for about $60 \%$ of the known periodic variable stars.

\section{Period Search: Approach II}

To increase the signal to noise ratio, the periodograms have been computed separately for the $B_{T}$ and $V_{T}$ bands with a final periodogram depending on both test functions. The main periodogram was computed based on a trigonometric polynomial fit of order 2 (TP2), of which the statistical response to noise is identical to the 5-bin PDM method. It allowed the recovery of periods of stars

\footnotetext{
${ }^{1}$ Department of Astronomy, Odessa State University, T. G. Shevchenko Park, UA-270014 Odessa, Ukraine

${ }^{2}$ Observatoire Astronomique de Strasbourg, 11 rue de l'Université, F-67000 Strasbourg, France

${ }^{3}$ Royal Observatory of Belgium, Ringlaan, 3, B-1180 Brussels, Belgium
} 
with smooth variations. For the stars with abrupt changes (e.g. EA, RR) we have used the weighted differences of the magnitudes in the methods of Lafler \& Kinman (1965) and Deeming (1970). Statistical properties of test functions of these and other non-parametric methods were recently studied by Andronov \& Chinarova (1997).

Among the 320 known periodic variables, the true periods were detected in the TP2 periodogram for 206 stars. In $38 \%$ of these cases it was the first or the second most significant peak. This indicates that the variations are often pronounced mainly in one color.

\section{Newly Developed Methods (Variable-Order Splines)}

The "RR-catcher" was used to find periods of stars with asymmetric one-wave light curves. We applied here a 5-parameter fit: the descending branch is fitted by a parabola, and the ascending branch by a cubic polynomial, keeping the smoothing curve and its first derivative continuous (see Andronov 1999, Marsakova \& Andronov 1996). For the "EA-catcher", the phase diagram is split into two minima of equal duration opposite in phase with an unknown depth, and a constant level outside minima. Both these methods need nonlinear 3D optimization for each candidate period. A comparison of the TP2, "RR-" and "EA-catcher" fits leads to changes in ranking the candidate periods, increasing reliability of the best period. For example, the residuals of the data from the best "EA-catcher" curve were smaller than those of the TP2 fit in $30 \%$ of stars.

\section{Conclusion}

We failed to find a single method able to derive reliable periods from the Tycho measurements. However, the most probable periods may be extracted by using several complementary period analysis methods.

A quick examination of the light curves of the possible new variable stars of our list revealed some good candidates of different types of variability, such as $\delta$ Scuti stars, RR Lyrae stars, and eclipsing binaries. However, additional observations are needed to confirm these results.

\section{References}

Andronov, I. L. 1999, Odessa Astron. Publ., 12 (in press)

Andronov, I. L. \& Chinarova L. L. 1997, Kinem. Phys. Cel. Bod., 13, 6, 55

Deeming, T. J. 1970, MNRAS, 147, 365

Halbwachs, J.-L. 1997 in The Hipparcos and Tycho Catalogues, ESA SP-1200, vol. 4 , ch. 9

Lafler J. \& Kinman T. D. 1965, ApJS, 11, 216

Marsakova V. I. \& Andronov I. L. 1996, Odessa Astron. Publ., 9, 127

Piquard, S., Halbwachs, J.-L., Fabricius, C., Geckeler, R., \& Wicenec, A. 2000, in these proceedings, p. 62

Stellingwerf, R. F. 1978, ApJ, 224, 953 